\title{
EHMTI-0056. Self-medication of headache: identification of subgroups of patients through cluster analysis
}

\author{
E Mehuys $^{1 *}$, K Paemeleire ${ }^{2}, \mathrm{G} \mathrm{Crombez}{ }^{3}$, T Van Hees ${ }^{4}$, T Christiaens $^{5}$, L Van Bortel ${ }^{6}$, I Van Tongelen ${ }^{1}$, JP Remon ${ }^{1}$, \\ K Boussery ${ }^{1}$
}

From 4th European Headache and Migraine Trust International Congress: EHMTIC 2014

Copenhagen, Denmark. 18-21 September 2014

\section{Introduction}

We have previously shown that medication overuse is prevalent among individuals self-medicating regular headache.

\begin{abstract}
Aims
In this study we evaluated self-medicating headache patients from a broader perspective, exploring the interplay between headache and concomitant pain conditions, pain-related disability and pain medication use. Identification of subgroups of patients could be helpful to tailor intervention strategies.
\end{abstract}

\section{Methods}

A hierarchical cluster analysis was used to group 1021 selfmedicating headache patients according to their (1) sociodemographics, (2) pain characteristics, (3) pain-related disability and (4) pain medication use. Patients were recruited in 202 Belgian community pharmacies and fulfilled the following inclusion criteria: aged $\geq 18$ years, purchasing an over-the-counter systemic analgesic, experiencing pain $\geq 1$ full day/month and suffering from headache.

\section{Results}

Three subgroups were identified. Group 1 comprised patients with low socioeconomic status, low self-rated health, on average four concomitant pain conditions, high pain frequency, high disability, and high rates of medication overuse. Group 2 included older patients with a mean of two other pain syndromes, and low disability but high pain intensity. Group 3 comprised young highly-educated patients diagnosed with migraine, having on average one concomitant pain condition, low pain frequency, low disability but high pain intensity, and low rates of medication overuse.

\section{Conclusions}

We have identified three subgroups in a large sample of individuals self-medicating headache. The marked differences across the three groups stress the importance of a holistic assessment of headache patients and the need for tailored strategies to reduce the risk of medication-overuse headache in primary care.

No conflict of interest.

\section{Authors' details}

${ }^{1}$ Pharmaceutical Care Unit, Ghent University, Ghent, Belgium. ${ }^{2}$ Department of Neurology, University Hospital Ghent, Ghent, Belgium. ${ }^{3}$ Department of Experimental Clinical and Health Psychology, Ghent University, Ghent, Belgium. ${ }^{4}$ Department of Clinical Pharmacy, University of Liège, Liège, Belgium. ${ }^{5}$ Department of Family Medicine and Primary Health Care, Ghent University, Ghent, Belgium. ${ }^{6}$ Heymans Institute of Pharmacology, Ghent University, Ghent, Belgium.

Published: 18 September 2014

doi:10.1186/1129-2377-15-S1-D46

Cite this article as: Mehuys et al:: EHMTI-0056. Self-medication of headache: identification of subgroups of patients through cluster analysis. The Journal of Headache and Pain 2014 15(Suppl 1):D46.

${ }^{1}$ Pharmaceutical Care Unit, Ghent University, Ghent, Belgium

Full list of author information is available at the end of the article 